\title{
La ville et son élan vital
}

Marcel Poëte et Robert Auzelle lecteurs de Bergson

Town and Vital Impetus. The interpretation of Bergson by Marcel Poëte and Robert Auzelle

Jean-François Guillot

\section{OpenEdition \\ Journals}

Édition électronique

URL : http://journals.openedition.org/crau/401

DOI : 10.4000/crau.401

ISSN : 2547-5746

Éditeur

Éditions du patrimoine

Édition imprimée

Date de publication : 1 décembre 2014

Pagination : 123-130

ISBN : 978-2-7577-0379-3

ISSN : 1296-4077

\section{Référence électronique}

Jean-François Guillot, «La ville et son élan vital », Les Cahiers de la recherche architecturale et urbaine [En ligne], 30/31 | 2014, mis en ligne le 14 septembre 2017, consulté le 03 mai 2019. URL : http:// journals.openedition.org/crau/401; DOI : 10.4000/crau.401 


\section{La ville et son élan vital. \\ Marcel Poëte et Robert Auzelle lecteurs de Bergson}

L'urbaniste doit, selon Robert Auzelle, "obéir à la poussée interne » ${ }^{1}$ qui est "dans une réalité humaine la manifestation de cet élan vital dont Bergson voyait la création parcourue tout entière. Marcel Poète a le premier emprunté à la philosophie bergsonienne ce terme pertinent et cette notion ». L'hommage d'Auzelle à son maître témoigne de la place centrale qu'occupe la philosophie bergsonienne dans la filiation ici reconnue.

Bien que contemporains, Marcel Poëte et Henri Bergson ne se sont probablement jamais rencontrés. Le philosophe a pourtant considérablement marqué le travail de l'historien des villes. L'importance du contexte bergsonien de la pensée de Poëte a été exposée dans les travaux de Steven Melemis ${ }^{2}$ et dans les écrits de Donatella Calabi ${ }^{3}$. Notre propos est de mettre un peu plus en lumière la manière dont Poëte a reçu la philosophie de Bergson en nous appuyant sur les résurgences de cette philosophie chez Auzelle. Bergson n'a jamais pris directement la ville comme objet d'étude. Sa pensée est une philosophie du vivant et du changement tant d'un point de vue de

1. Robert Auzelle, Plaidoyer pour une organisation consciente de l'espace, Paris, Vincent, Fréal \& Cie, 1962, p. 35

2. Steven Melemis, « Marcel Poëte précurseur », in Colonnes, n० 19, novembre 2002, p. 26.

3. Donatella Calabi, Marcel Poëte et le Paris des années vingt, Paris, L'Harmattan, 1998, p. 69-77. 
l'évolution naturelle que de la liberté humaine dans l'agir individuel et social. C'est bien une transmutation des idées bergsoniennes qui s'opère dans la pensée de Poëte pour former et exprimer une histoire des villes non seulement avec un vocabulaire bergsonien, mais aussi par le renouvellement de la méthode historique à l'aune de la philosophie de l'évolution et de la durée.

Robert Auzelle utilise régulièrement le vocabulaire bergsonien dans son œuvre. Souvent il s'agit de simples métaphores, mais ses textes montrent aussi que la philosophie de Bergson faisait partie de la culture de cet urbaniste. Poëte n'a bien sûr pas fait découvrir Bergson à Auzelle, mais la marque de l'enseignement de Poëte dans le bergsonisme d'Auzelle se fait jour lorsqu'on compare les écrits de l'historien et ceux de l'urbaniste. La qualité de disciple de Marcel Poëte que Robert Auzelle revendiquait a été étudiée par Frédéric Bertrand ${ }^{4}$. Nous souhaitons ici mettre l'accent sur la place de la philosophie de Bergson dans cette transmission.

Bergson, Poëte et Auzelle furent des penseurs spiritualistes et chrétiens, chacun à sa manière. Mais l'objet de cette étude n'est pas tant de leur chercher des points communs que de suivre le parcours singulier des idées du philosophe chez ces deux figures majeures de I'urbanisme. Des notions fondamentales de la philosophie de Bergson suivent en effet une aventure depuis les lectures qu'en fit Poëte jusqu'aux enseignements et aux mises en œuvre d'Auzelle. Ces notions, toujours liées, très fréquemment méditées par Poëte et souvent utilisées par Auzelle, sont la durée et l'intuition. Elles ont mené Poëte à l'idée d'âme urbaine, qu'il a travaillée jusqu'à la fin de sa vie. C'est une transposition de l'âme urbaine selon Poëte qui apparaît chez le praticien de la ville qu'a été Auzelle.

Depuis l'enseignement de Poëte avant la Deuxième Guerre mondiale jusqu'aux travaux et aux écrits d'Auzelle dans le contexte de la Reconstruction et au-delà le parcours des idées issues du bergsonisme est difficile à suivre, car il s'agit d'idées vivantes au sens où l'entendait Bergson: la marque de leur authenticité est qu'elles se transforment avec ceux qui les pensent.

\section{La durée comme composition de la ville}

Poëte note que l'élan vital selon Bergson produit un organisme "fait de différences multiples et complémentaires [...]. Bergson en parle dans le sens de la création, par la nature, de l'espèce humaine. Mais cela peut jusqu'à un certain point s'appliquer aux étapes de la création de l'espèce urbaine ${ }^{5}$ ». Chez Bergson en effet, la durée permet de rendre compte de l'évolution du vivant qui n'est pas créé selon un plan mais qui s'invente sans cesse à partir de son passé: "Durée signifie invention, création de formes, élaboration continue de l'absolument nouveau ${ }^{6}$." Plus précisément, chez le philosophe, l'évolution est créatrice parce que l'invention consiste à se détacher d'une forme existante pour en créer une nouvelle qui correspondra à une nouvelle fonction. Durer, créer, c'est diviser. Une telle division se réalise exactement dans ce que Poëte nomme " espèce urbaine » et qui se manifeste par l'exemple qu'il reprend souvent de la création de l'université de Paris. Au fil des réécritures, I'histoire de la naissance de l'université devient de plus en plus une "évolution créatrice " au sens où l'entend
4. Frédéric Bertrand, "La nature du cimetière moderne, hypothèses autour de l'œuvre de Robert Auzelle (1913-1983) », EAV (Enseignement architecture ville), n० 15, 2010, p. 70.
5. Marcel Poëte, MS 209 (4) [les manuscrits de Poëte sont conservés à la Bibliothèque historique de la Ville de Paris].

6. Henri Bergson, L'Évolution créatrice, Paris, PUF, 1940, p. 11. 
Bergson. En effet, la division est d'abord une double orientation partant de l'île de la Cité qui ne peut plus contenir à elle seule toutes les fonctions de la ville: le Grand-Pont dirige Paris vers le nord sur la route du commerce, tandis que le Petit-Pont ouvre la Cité vers les centres intellectuels et spirituels que sont Orléans et le pèlerinage de Saint-Jacques. C'est de cette division qu'est née l'université: "En se déplaçant de l'île de la Cité à cette partie méridionale de Paris à laquelle conduit le Petit-Pont, le mouvement des études dans cette ville a conquis sur l'évêque de Paris et sur le chapitre de Notre-Dame, cette sorte d'autonomie qu'est l'université?. "

Pourtant cette séparation, même si elle est une indépendance gagnée par l'université aux dépens de l'évêque, conserve la présence de son origine: les écoles sont poussées "hors de la cathédrale qui leur avait donné naissance pour faire de la rive gauche I'université ${ }^{8} »$. Au fil du temps, Poëte insiste davantage sur l'unité dans la diversité et dans la création: "On part de la dispersion pour aboutir, au long des âges, à I'unification'. " On retrouve là I'une des originalités de la pensée de Bergson: les divisions ne sont pas sans mémoire: les termes extrêmes de l'évolution que sont l'homme et les insectes ont conservé une origine commune, c'est pourquoi le travail des abeilles n'est pas dénué d'intelligence, et, réciproquement, l'intelligence de l'homme ne s'est pas totalement défaite de I'instinct qui commande la vie sociale des insectes ${ }^{10}$. Le processus de division assure l'unité de l'ensemble du vivant, car la création nouvelle a toujours tendance à s'associer aux autres formes créées.
Auzelle considère la ville d'une façon analogue. Mais, tandis que Poëte décrit une évolution presque spontanée du Paris médiéval, I'urbaniste a en charge des projets. Le défi de la valorisation du temps apparaît d'abord a contrario lorsqu'Auzelle parle du goût des commanditaires pour l'éternel et l'immuable, ce goût vient contredire l'élan vital: "Les puissants de ce monde ne se voient pas vieillir, ils ne voient pas le monde à peine saisi leur échapper et ils attendent de I'architecte des bâtiments qui figeraient une fois pour toutes une multitude de fonctions, - lesquelles pourtant ne peuvent que résulter du jaillissement complexe, et toujours renouvelé de la vie collective ${ }^{11}$. " Le reproche est le même que celui que faisait Bergson à nombre de philosophes, constatant avec regret "qu'aucun d'eux n'a cherché au temps des attributs positifs. Ils traitent la succession comme une coexistence manquée, et la durée comme une privation d'éternité. De la vient qu'ils n'arrivent pas, quoi qu'ils fassent, à se représenter la nouveauté radicale et l'imprévisibilité ${ }^{12} »$.

Pour Auzelle, "la ville est un être vivant; plus qu'une analogie, c'est une réalité, combien difficile à saisir, certes, et encore plus à créer. Mais il n'est pas interdit, philosophiquement parlant, de s'aider de l'analogie lors de cette gestation, à condition de garder constamment à l'esprit le contact avec la vie, celle des hommes qui font la ville, donc celle de la ville ${ }^{13} »$. Auzelle reconnaît comme Poëte la société urbaine inscrite dans une histoire comme le matériau premier de la ville et de sa durée. Comme pour I'historien, la philosophie du vivant est pour Auzelle une attitude de l'esprit, mais elle ne constitue pas à elle seule le métier de l'urbaniste: "Ce contact exige la connais-
7. M. Poëte, Une vie de cité, Paris de sa naissance à nos jours, 3 tomes et un album, Paris, Picard, 1924-1931, t. I, p. 163-164.

8. M. Poëte, «Les idées bergsoniennes et l'urbanisme » in Mélanges à Paul Négulesco, Bucarest, Imprimeria Nationala, 1935, p. 575-585, [art. cité intégralement en annexe de Rémi Baudoui, La Naissance de I'École des hautes études urbaines et les premiers enseignements de l'urbanisme en France des années 1910 à 1920 (Paris, école d'architecture Paris-Villemin/Ardu, Paris 8, 1988) auquel on se réfère ici, p. 166].

9. M. Poëte, Une vie de cité, op. cit. note 7, t. I, p. 256

10. H. Bergson, L'Évolution créatrice, op. cit. note 6, p. 126 et 259 .

11. Ibid.
12. H. Bergson, La Pensée et le mouvant, Paris, PUF, 1941, p. 10

13. R. Auzelle et al., Trois cent vingt-trois citations sur l'urbanisme, Paris, Vincent, Fréal \& Cie, 1964, p. 764-765.

14. M. Poëte, MS 216 (3), fol. V. 
sance, et impose l'analyse qui, pour être complète doit se ramifier jusqu'à chaque phénomène élémentaire. " L'idée de ramification se comprend par analogie avec la durée des espèces vivantes lors de l'évolution. Sans doute Auzelle pense-t-il à une image de Bergson plusieurs fois reprise par Poëte à propos de Paris: "La fusée de la vie dissocie les fonctions urbaines, originellement localisées dans l'île ${ }^{14}$. » Dans le texte cité plus haut, Auzelle constate que, dans la pratique, on peut rejoindre la durée de la ville: « II est alors frappant de constater que le résultat de cette analyse conduit à la même conclusion que l'approche métaphysique du problème; on est fondé à trouver dans cette convergence une preuve de la validité de la thèse ici soutenue. " Mais c'est bien l'urbaniste qui a accès à l'élan vital, pas l'architecte: c'est à cause de la composition que «nous [architectes] soutenons que, de toutes les disciplines, l'urbanisme est celle qui nous prépare le mieux à notre profession ${ }^{15}$ ». La composition statique est légitime dans tous les arts, mais en architecture c'est une tendance regrettable que l'urbaniste peut rectifier s'il apporte l'élan de la composition dynamique.

C'est justement à travers la notion de composition que Poëte montre comment Paris est sorti de l'urbanisme naturel au XVII siècle. II a fallu attendre les travaux de Le Nôtre aux Tuileries, pour que naisse la création urbaine véritable. Poëte s'en explique ainsi : "On ne lui [Le Nôtre] doit point de formes nouvelles à proprement parler. Les éléments du jardin à la française existaient ${ }^{16}$ ", la nouveauté est dans la création d'un nouvel ensemble qui transforme l'idée de ville: "N'est-ce là toutefois qu'un simple jardin? [...] II faut considérer un tel lieu comme une véritable composition urbaine, au même titre qu'une place de ville par exemple »; plus loin il ajoute en comparant le travail de Le Nôtre à celui de ses prédécesseurs: «Bref, un assemblage a fait place à une composition, celle-ci englobant dans un tout l'édifice et le paysage [...] c'est une composition urbaine comme la place du Capitole et la place Saint-Pierre à Rome. »

Passer de l'assemblage à la composition autorise la durée authentique. Le quartier de la Plaine à Clamart, conçu par Auzelle, est une démonstration de l'urbanisme de composition et non d'assemblage, ouvert à la transformation et à l'imprévisible dans la mesure où l'urbaniste a choisi, par la variété de hauteur et de disposition des constructions, de ne pas bloquer l'avenir de sa propre réalisation. C'est une œuvre urbaine conforme à sa méthode, où "les bâtiments doivent eux-mêmes convenir au terrain qu'on leur destine, s'accommoder les uns des autres dans leur voisinage réciproque et se mesurer heureusement avec le " mobilier vide» (plantations, bas édifices, etc.) ${ }^{17}$ ". La preuve de la réussite de cette composition est donnée par Gérard Thurnauer qui, avec l'Atelier de Montrouge, a construit la bibliothèque pour Enfants au sein du quartier de la Plaine dans des formes et avec un matériau tout différents de ceux employés jusque-là.

C'est en observant la cité de la Plaine que l'on peut comprendre cette pensée toute bergsonienne d'Auzelle : "L'avenir que nous concevons doit, perpétuellement, contenir l'avenir ${ }^{18}$. " L'urbaniste a en charge la durée de la ville qui fait pièce à l'illusion d'éternité des urbanistes et des commanditaires soucieux de la permanence. La lecture de Poëte montre que le Paris du Moyen Âge n'avait pas le souci de sa conservation du fait de son
15. R. Auzelle, L'Architecte, Paris, Vincent \& Fréal, 1965, p. 106
16. M. Poëte, Au jardin des Tuileries. L'art du jardin, la promenade publique, Paris, Picard, 1924 , p. 246. Voir également MS 127, p. 108 et MS 209 (4)
17. R. Auzelle, Trois cent vingt-trois citations..., op. cit. note 13, p. 661.

18. R. Auzelle, « Les grandes villes et I'urbanisation de l'avenir », Urbanisme, $n^{\circ} 102-103,1967$, p. 11.

19. H. Bergson, Essai sur les données immédiates de la conscience, Paris, PUF, 1940, p. 75 
accroissement quasi naturel. Pourtant, chez l'historien comme chez l'urbaniste, la durée de la ville est une durée de création. Percevoir ou créer une durée nécessite I'usage de l'intuition, faculté originale et fondamentale dans la philosophie de Bergson, mise en œuvre de façon particulière par Auzelle et par Poëte.

\section{L'intuition pour connaître et créer la ville}

L'idée de durée apparaît chez Bergson d'abord au point de vue psychologique: tout le passé de l'individu ne cesse de se transformer au contact du monde présent. Le moi présent porte en lui-même un passé mobile. Ainsi notre passé n'est pas divisible en étapes, nos états de conscience s'organisent, «comme il arrive quand nous nous rappelons, fondues pour ainsi dire ensemble, les notes d'une mélodie ${ }^{19}$ ". L'expérience de la durée psychologique a conduit Bergson à l'identification de l'intuition comme faculté de connaître ce qui est essentiellement mobile. L'intuition se distingue de l'intelligence qui nous permet de nous représenter le monde, de le transformer, mais en nous détachant de la réalité mouvante pour y découper des idées aux contours fixes. À l'inverse, l'instinct des animaux les fait adhérer à la vie en mouvement; mais l'instinct ne leur permet aucun recul, aucune liberté par rapport à l'élan de la vie dans lequel ils sont comme engloutis. L'intuition offre à I'homme un mode unique de connaissance, elle est « un instinct désintéressé, conscient de lui-même, capable
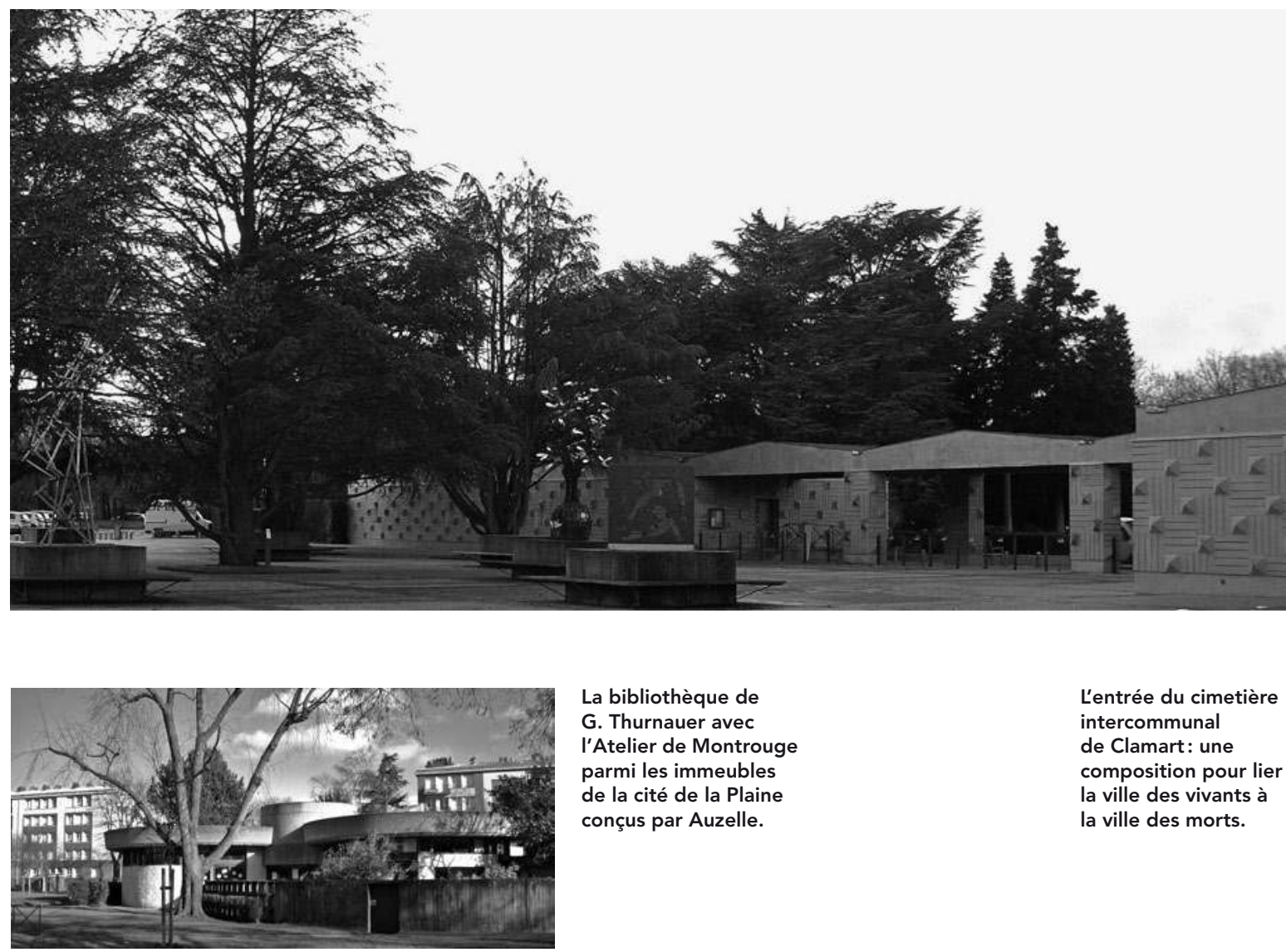

La bibliothèque de G. Thurnauer avec l'Atelier de Montrouge parmi les immeubles de la cité de la Plaine conçus par Auzelle.
L'entrée du cimetière intercommunal de Clamart: une composition pour lier la ville des vivants à la ville des morts. 
de réfléchir sur son objet et de l'élargir indéfiniment ${ }^{20} »$. La réalité du monde est durée, mouvement continuel, elle est inaccessible à l'intelligence calculatrice mais se dévoile à l'intuition pour qui accepte d'entrer dans l'élan vital des choses.

L'intuition comme méthode de l'historien a préoccupé Poëte des années 1930 à la fin de sa vie, elle va à contrecourant de l'intelligence qui "remonte » le courant de I'histoire des effets aux causes. "Écoulement incessant des choses, lien de tout à tout, continuité de la vie de la ville: voilà des idées générales dont il faut être pénétré pour l'étude de l'évolution urbaine ${ }^{21}$. " La durée de la ville n'est accessible qu'à celui qui se reconnaît d'abord lui-même comme un être dans la durée. C'est le cas d'Auzelle qui appréhende la ville d'une manière analogue et tout aussi bergsonienne lorsqu'il écrit que "I'histoire, qui est mémoire d'un devenir, apporte des éléments d'information par lesquels s'expliquent ou se justifient telles formes actuelles de l'occupation d'un territoire ${ }^{22} »$. Mais la trace de l'enseignement bergsonien de Poëte est plus lisible encore quand Auzelle écrit qu'il faut faire œuvre "d'artiste sensible " pour "dégager de l'évolution du paysage et de l'architecture à travers les âges ce qui fait le caractère propre de l'agglomération ${ }^{23} »$.

L'intuition bergsonienne est en effet plus proche du geste créateur de l'artiste que de la connaissance, car la création accompagne le mouvement de la vie. "L'intention de la vie, écrit Poëte, qui échappe à notre œil, l'artiste vise à la ressaisir en se replaçant à l'intérieur de l'objet par une espèce de sympathie ${ }^{24}$. " Plus qu'une condition de la création, l'intuition se confond avec celle-ci, car elle révèle l'intériorité du mouvement de la vie dans lequel tout artiste doit s'inscrire. Ainsi en va-t-il de l'artiste-urbaniste selon Poëte: "Réaliser ou maintenir l'adaptation de la ville au site, tirer parti des différents niveaux, [...] sauvegarder et utiliser les points de vue, constituent pour un urbaniste avisé, des moyens propres à faire de la ville plus qu'une machine à vivre: une œuvre $d^{\prime} a r^{25}$. » C'est à cette injonction qu'Auzelle veut obéir en faisant de la disposition d'esprit de l'urbaniste le pendant de celle que Poëte exige de l'historien: "Les yeux clos tout au fond de l'être, le passé alors se confond avec le présent. Ainsi se nouent les liens étroits entre la ville et l'homme qui tente de la comprendre ${ }^{26}$. " Le principe de la connaissance que doit avoir l'urbaniste selon Poëte est parfaitement bergsonien. L'urbaniste est un artiste et la ville une œuvre d'art, car dans l'émotion esthétique il n'y a qu'une seule intuition, partagée par l'artiste et le spectateur. En somme, I'historien doit être d'abord le spectateur authentique de la ville dans sa durée et l'urbaniste véritable doit être comme les grands qui ont transformé les sociétés en saisissant le fond mouvant de leur être. Poëte écrit que la forme urbaine devenue œuvre d'art influe sur l'être « du fait que, comme l'a écrit Bergson, l'artiste est un de ces privilégiés en qui s'est dilatée l'âme sociale ${ }^{27}$ ".

Dans la même attitude de pensée, Auzelle exprime ce que Bergson et Poëte nomment intuition: "Si I'œil reçoit le monde et le transmet, c'est l'esprit qui, armé de toute l'expérience acquise, voit véritablement ${ }^{28}$." Cependant, à la différence de Poëte, Auzelle ancre moins explicitement sur le vivant la méthode de connaissance de l'urbaniste. En revanche, quant à l'urbaniste comme artiste du temps, Auzelle le veut à l'œuvre avec tout
20. H. Bergson, L'Évolution créatrice, op. cit. note 6, p. 178.

21. M. Poëte, "Évolution du plan des villes", in Bulletin du Comité international des sciences historiques, n² 20, juillet 1933, p. 537.

22. R. Auzelle, Plaidoyer pour une organisation consciente de l'espace, op. cit. note 1, p. 60.

23. R. Auzelle, Cours d'urbanisme à I'Institut d'urbanisme de l'université de Paris.
Première année. Intelligence du milieu et stratégie de l'aménagement, t. I, Paris, Vincent, Fréal et $C^{i e}, 1967$, p. 86

24. M. Poëte, MS 209 (2), fol CC.

25. M. Poëte, « Des plans d'aménagement des villes », in Bibliothèque de l'Institut des sciences administratives de Roumanie, $n^{\circ} 35$; extrait de la Revista de drept public, 5 e année, nos 3-4, Bucarest, 1931, p. 12.
26. M. Poëte, MS 122, p. 299.

27. M. Poëte, «Urbanisme », Urbanisme, no 63, 1938, p. 146. Sur la notion de dilatation, Poëte se réfère à $\mathrm{H}$. Bergson, Les Deux Sources de la morale et de la religion, Paris, PUF, 1941, p. 74-75.

28. R. Auzelle, L'Architecte, op. cit. note 15, p. 40 
son être dans la vie urbaine: "L'art de l'environnement, nous ne nous avisons pas de le désigner comme un Être distinct: tant il se distingue peu de notre vie ${ }^{29}$ ! " Sans la nommer « intuition", c'est bien à cette faculté que fait appel Auzelle quand il écrit que «le site est une ébauche naturelle [...]. Les circonstances les plus diverses s'allient pour constituer un écrin en perpétuel devenir. Le temps est son ami ou son ennemi suivant que l'on a fait preuve de clairvoyance ou de pusillanimité, qu'on a emprisonné ou ménagé son avenir, écrasé ou préparé les extensions futures ${ }^{30}$ ». L'urbaniste doit confondre son être propre avec l'être du site urbain, non seulement pour laisser la place à des extensions à venir, mais pour créer de la ville qui offrira à son tour la possibilité de nouvelles naissances encore imprévisibles. L'apport du bergsonisme de Poëte chez Auzelle est moins une approche de la spécificité du vivant que la prise de conscience de l'intimité que doit avoir l'urbaniste avec l'être de la ville sur laquelle il travaille, cet être étant fait de la présence mobile de tout son passé. Seule l'adhésion à un tel mouvement peut garantir l'urbaniste contre la réalisation d'œuvres mortes, c'està-dire sans avenir. Frédéric Bertrand a montré l'importance de l'anonymat de l'œuvre et la supériorité de la composition par rapport à une "addition d'individus" 31. Un tel anonymat, vu sous l'angle de la philosophie bergsonienne, n'est pas une vertu morale de l'urbaniste, c'est une condition de la composition véritable dans la durée dont la source est l'union intuitive de I'artiste et de la ville dans laquelle il travaille. L'élan vital est commun à l'urbaniste et à son œuvre qui, de ce fait, lui échappe.
Dans un court dialogue à la manière de Paul Valéry dans Eupalinos, Auzelle met en scène un jeune architecte et Socrate. Le philosophe, ayant conduit son disciple jusqu'aux Lois et jusqu'aux principes de la Géométrie, entend le jeune architecte constater que tout cela était adapté «à un monde que nous pensions incomparable », tandis qu'il doit travailler « au milieu d'un monde plus incertain que la mer». Socrate répond: "Oui... mais le propre de l'homme, depuis l'origine, n'est-il pas d'être au bord même de l'avenir ${ }^{32}$ ? " C'est bien " au bord de l'avenir » qu'il faut se tenir pour ne pas succomber à l'illusion de l'éternité comme possession figée de I'avenir, travers dû tant à la vanité des commanditaires qu'à la tendance naturelle que Bergson attribue à l'intelligence éloignée du mouvement de la vie.

\section{"L'âme urbaine » et la ville libre}

Une ville se développant sans le secours de la composition urbaine se condamne à rester ou à redevenir une société close au sens où l'entend Bergson, c'est-à-dire une société régie par l'obéissance comme garant de la conservation. À l'inverse, selon le philosophe, la société ouverte est celle où celui qui a "dilaté en lui l'âme sociale" ne se contente pas d'un «nouvel arrangement de l'ancien » et sait répondre à une "exigence de création ${ }^{33}$ ". Celui-là entraîne avec lui l'ensemble de la société sur une voie radicalement neuve. C'est donc la liberté qui est en jeu dans le développement de la ville.

Poëte s'interroge sur la société et la ville industrielles qui n'ont pas encore trouvé de créateurs urbains malgré l'élan de liberté apporté par le machinisme: «Le règne de la machine libératrice de l'humanité dans idée de
29. R. Auzelle, "Les grandes villes et l'urbanisation de l'avenir », Urbanisme, $n^{o s} 102-103,1967$, op. cit., p. 11

30. R. Auzelle, Plaidoyer pour une organisation consciente de l'espace, op. cit. note, p. 84

31. F. Bertrand, « Robert Auzelle et

I'urbanisme français des années cinquante », Urbanisme, n 307, août 1999, p. 43.
32. R. Auzelle, L'Architecte, op. cit. note 15, p. 107.

33. H. Bergson, Les deux sources de la morale et de la religion, p. 43-44. 
Bergson. Le mysticisme ou la poésie de la machine ainsi conçue. Haut les cœurs devant les lignes montantes de la tour Eiffel ${ }^{34}$ ! » À l'époque où travaille Auzelle, la question du machinisme n'a plus la même acuité. C'est donc sur un autre plan qu'il envisage l'opposition entre ville statique et ville créatrice, entre le clos et l'ouvert ${ }^{35}$, mais l'origine de cette césure est aussi nettement bergsonienne que chez Poëte, et c'est la composition qui partage les deux attitudes: « Là où la vie est intense, l'architecte "pétrifié » intervient avec des soucis d'un autre âge, les pensées et les dogmes d'une époque révolue. Le principe même de son art, il l'ignore: il ignore que l'art de la composition est consubstantiel à sa profession, comme il l'est à la vie elle-même ${ }^{36}$. " II incombe à l'urbaniste de ne pas chercher à soustraire son œuvre au temps, condition paradoxale pour assurer la continuité de création de la ville avec et après lui.

Le devoir d'assumer la temporalité et de faire un avenir qui "contienne l'avenir», pour que la ville ne soit pas close, trouve chez Auzelle une expression singulière dans l'insistance avec laquelle il prône une intégration des nécropoles dans le tissu urbain. Pour le comprendre, il faut se garder de découper les travaux d'Auzelle en "compartiments étanches ${ }^{37}$ » et de voir d'un côté l'urbaniste et de l'autre le "spécialiste du funéraire ». L'attention portée aux morts est le signe d'une durée urbaine assumée. Toute séparation dans l'esprit de l'urbaniste entre la ville des vivants et la nécropole serait une rupture de l'élan vital, une manière de nier la durée qui est un passé perpétuellement présent. Auzelle note que "lorsqu'un homme meurt, la société ne perd pas seulement une unité, elle perd un être social ${ }^{38}$ ». Est «social » ce qui fait partie de la durée de la ville, de ce que Poëte approchait avec I'idée d'âme urbaine. C'est pourquoi, selon Auzelle, « le cimetière fait partie de l'existence des sociétés. Il est un des liens entre le passé et le présent; il inscrit dans la terre la continuité des générations, leur solidarité, leurs mystérieux échanges; il est, qu'on le veuille ou non, une forme du sacré, et comme une métaphysique matérialisée ${ }^{39}$ ». Sans la matérialisation de cette métaphysique de la durée, la ville pourra s'agrandir mais elle restera une société close, "pétrifiée» dans une illusion d'éternité.

L'une des réalisations les plus significatives de l'attention aux morts pour assurer la durée de la ville et son élan vital est le cimetière intercommunal de Clamart. Ce cimetière paysager exalte la vie tout en favorisant un paisible recueillement. Le mur du cimetière est le contraire d'une séparation entre vivants et morts puisqu'il ne suit pas de ligne droite et il est comme traversé par un alignement d'arbres, cette composition est une scénographie du vivant, une " matérialisation de la métaphysique » que prône Auzelle. Toutefois celui-ci aurait aimé plus de continuité avec la « ville des vivants ", mais les édiles n'ont pas suivi ses préceptes ${ }^{40}$.

La tragédie de la ville froidement intelligente est qu'elle simule l'éternité, oublieuse qu'elle est de "l'âme urbaine " qui est une histoire avec laquelle l'urbaniste doit faire corps, de même que l'historien des villes ne doit faire qu'un avec son objet dans l'intuition créatrice porteuse de l'élan vital et nourrie par lui.
34. M. Poëte, MS 209 (4).

35. Voir F. Bertrand, « Robert Auzelle et I'urbanisme français... ", op. cit. note 4, p. 43.

36. R. Auzelle, L'Architecte, op. cit. note 15, p. 106.

37. F. Bertrand, «Une exposition et des rencontres", Colonnes, n० 19 , novembre 2002, p. 5.
38. Fonds Robert Auzelle, Siaf/Cité de l'architecture et du patrimoine/Archives d'architecture du xx siècle, $n$. № 242 IFA 2/ RAL.

39. R. Auzelle, Dernières Demeures. Conception, composition, réalisation du cimetière contemporain, Paris, 1965, p. 404.
40. Fonds Robert Auzelle, Siaf/Cité de I'architecture et du patrimoine/Archives $d^{\prime}$ architecture du $x x^{e}$ siècle, $n^{\circ} 242$ IFA 5/RA 13 et $n^{\circ} 242$ IFA 4/RA 05. 